\title{
Relationships between apparent diffusion coefficient (ADC) histogram analysis parameters and PD-L 1-expression in head and neck squamous cell carcinomas: a preliminary study
}

\author{
Hans-Jonas Meyer ${ }^{1}$, Anne Kathrin Höhn², Alexey Surov³ \\ ${ }^{1}$ Department of Diagnostic and Interventional Radiology, University of Leipzig, Leipzig, Germany \\ 2 Department of Pathology, University of Leipzig, Leipzig, Germany \\ ${ }^{3}$ Department of Diagnostic and Interventional Radiology, University of Magdeburg, Germany
}

Radiol Oncol 2021; 55(2): 150-157.

Received 22 October 2020

Accepted 13 December 2020

Correspondence to: Hans-Jonas Meyer M.D., Department of Diagnostic and Interventional Radiology, University of Leipzig, Germany. E-mail: hans-jonas.meyer@medizin.uni-leipzig.de

Disclosure: No potential conflicts of interest were disclosed. All authors contributed equally to this work

Background. Immunotherapy has become a cornerstone of the modern cancer treatment. It might be crucial to predict its expression non-invasively by imaging. The present study used diffusion-weighted imaging (DWI) quantified by whole lesion apparent diffusion coefficient ( $A D C$ ) values to elucidate possible associations with programmed cell death ligand 1 (PD-L1) expression in head and neck squamous cell cancer (HNSCC).

Patients and methods. Overall, 29 patients with primary HNSCC of different localizations were involved in the study. DWI was obtained by using a sequence with b-values of 0 and $800 \mathrm{~s} / \mathrm{mm}^{2}$ on a 3 T MRI. ADC values were evaluated with a whole lesion measurement and a histogram approach. PD-L1 expression was estimated on bioptic samples before any form of treatment using 3 scores, tumor positive score (TPS), immune cell score (ICS), and combined positive score (CPS).

Results. An inverse correlation between skewness derived from ADC values and ICS was identified $(r=-0.38, p=$ 0.04). $A D C_{\text {max }}$ tended to correlate with ICS $(r=-0.35, p=0.06)$. Other ADC parameters did not show any association with the calculated scores.

Conclusions. There is a weak association between skewness derived from ADC values and PD-L1 expression in HNSCC, which might not be strong enough to predict PD-Ll expression in clinical routine. Presumably, ADC values are more influenced by complex histopathology compartments, comprising cellular and extracellular aspects of tumors than only of a single subset of tumor associated cells.

Key words: head and neck squamous cell cancer; apparent diffusion coefficient; diffusion weighted imaging; programmed cell death ligand 1

\section{Introduction}

Head and neck squamous cell carcinoma (HNSCC) is one of the most frequent malignancies. ${ }^{1}$ Modern functional imaging modalities, comprising diffusion-weighted imaging (DWI) and Dynamic- contrast enhanced MRI provide further insight into tumor microstructure. ${ }^{2}$ With these approaches, several diagnostic and prediction aspects were made possible, including the prediction of several histological features in tumors, treatment success and new prognostic..$^{2-5}$ 
DWI assesses random-water motion in tissues, namely Brownian molecular movement and can be quantified by apparent diffusion coefficient (ADC). ${ }^{3,5}$ This water movement is hindered predominantly by cellularity and thus it is a wellknown fact that ADC values are inversely correlated with cell count with yet different results across several tumor entities. ${ }^{5}$ However, ADC values are not only associated with cellularity, but also with other histopathologic features, such as proliferation potential, neoangiogenesis, tumor invading lymphocytes or extracellular matrix. This was also shown for HNSCC. ${ }^{6-9}$

Recently, various studies elucidated further associations of ADC values and tumor characteristics in oncology. ADC values aid in diagnostic purposes, as benign tumors have significantly higher ADC values than malignant tumors. This was also reported for head and neck lesions. ${ }^{10-12}$ Another important aspect is treatment prediction. A higher pretreatment ADC value and, therefore, presumably a tumor with a lower cell density and a less malignant tumor biology, is an independent favorable prognostic factor to chemotherapy and radiochemotherapy. ${ }^{13-15}$ Moreover, ADC values are independently associated with overall prognosis. ${ }^{13-15}$

There is a growing interest in targeting immune modulatory checkpoints, especially the programmed cell death protein 1 (PD-1) around oncology. ${ }^{16}$ It is a transmembrane protein that is frequently expressed on the surface of T-lymphocytes.

TABLE 1. Overview of the included patients

\begin{tabular}{lc}
\hline Localization & $\mathbf{n}(\%)$ \\
\hline Tonsil & $7(24.1)$ \\
Tongue & $7(24.1)$ \\
Hypopharynx & $6(20.7)$ \\
Oropharynx & $5(17.2)$ \\
Larynx & $4(13.9)$ \\
\hline Grading & $12(37.5)$ \\
\hline Moderate (G2) & $17(62.5)$ \\
\hline Poor (G3) & $6(20.7)$ \\
\hline Stage & $10(34.5)$ \\
\hline T2 & $13(44.8)$ \\
\hline T3 & $25(86.2)$ \\
\hline T4 & $4(13.8)$ \\
\hline Nodal status &
\end{tabular}

Programmed cell death ligand 1 (PD-L1) is a transmembrane protein, which is physiologically expressed at low levels in several cells, such as vascular, endothelial and immune cells. Interestingly, it can be highly expressed in neoplastic cells. ${ }^{16}$ The binding of PD-L1 to PD-1 forms an immunological situation that can impair the proliferation and the function of the immune cells against the tumor. That is why high expression of PD-L1 enables cancer cells to escape from immunologic response. ${ }^{16}$

Immunotherapy was stated as a new paradigm shift in HNSCC treatment. ${ }^{17}$ Moreover PD-L1 expression on immunohistochemical specimen was shown to predict progression free survival in in advanced HNSCC stages and can predict metastases. ${ }^{18-20}$

However, it is unclear, whether ADC values derived from MRI are associated with the amount of PD-L1 stained tumor cells and the associated PD-L1 stained immune cells and not only with the whole tumor cellularity of the lesion, which might not be as clinically relevant as to predict several subtypes of cells.

Therefore, the present study sought to elucidate possible associations between ADC values derived from MRI and the amount of PD-L1 stained tumor parts including tumor cells and immune cells within the tumor.

\section{Patients and methods}

This study is a retrospective analysis of a prospectively acquired patient sample of consecutive HNSCC patients undergoing MRI evaluation for staging purposes. It was approved by the institutional review board (Ethic committee of the University of Leipzig, study codes 180-2007, 20110-12072010, and 341-15-05102015) and every patient gave their written consent.

\section{Patients}

Overall, 29 patients with primary HNSCC of different localizations were involved in the study. There were 8 (27.6\%) women and 21 (72.4\%) men with a mean age of $55.2 \pm 10.8$ years, range $33-77$ years. The patients received no specific treatment before the MRI.

Table 1 gives an overview of the included patients. In 12 patients (37.5\%) moderately differentiated tumors (G2), and in 17 cases (62.5\%) poorly differentiated carcinomas (G3) were diagnosed. The identified tumors were staged as T2 in 6 pa- 
tients $(20.7 \%)$, T3 in 10 patients $(34.5 \%)$, and as T4 in 13 cases $(44.8 \%)$. Most patients $(n=25,86.2 \%)$ had nodal metastases.

\section{DWI}

DWI was obtained by using an axial EPI (echo planar imaging) sequence with b-values of 0 and $800 \mathrm{~s} /$ $\mathrm{mm}^{2}$ (TR/TE: $8620 / 73 \mathrm{~ms}$, slice thickness $4 \mathrm{~mm}$, and voxel size $3.2 \times 2.6 \times 4.0 \mathrm{~mm}$ ) on a $3 \mathrm{~T}$ MRI scanner (Siemens Biograph mMR; Siemens Healthcare, Erlangen, Germany) within a routinely acquired MRI protocol. ADC maps were automatically generated by the implemented software. DWI data was processed with a custom-made Matlab-based application (The Mathworks, Natick, MA, USA). On the ADC maps a volume of interest was manually drawn at tumor boundary in accordance to the T1-weighted contrast enhancing tumor areas using all slices, as performed before. ${ }^{8,9}$ All measures were performed by one experienced author (AS, 16 years of general radiological experience and 6 years of experience on the field of head and neck radiology) blinded to the histopathology results. The following histogram parameters were calculated: mean, maximum, minimum, median, mode, $10^{\text {th }}, 25^{\text {th }}, 75^{\text {th }}$ and $90^{\text {th }}$ percentile as well as kurtosis, skewness and entropy.

\section{PD-L1 expression analysis}

The histopathology analysis was performed by an experienced board-certified pathologist blinded to the imaging results. PD-L1 expression was retrospectively evaluated by using an immunohistochemistry (IHC) assay, a routinely used version of PD-L1 (SP142) IHC assay (Roche Diagnostics, 68305 Mannheim, Germany). The staining protocol used in this study was as described in the instructions for the approved commercial assay. The assay was used to determine PD-L1 expression on tumor samples derived from clinical pretherapeutic bioptic specimen of the primary tumor. The biopsy was performed before the MRI within a short time interval of a few days. Three scoring methods were used to assess the immunohistochemical expression. Firstly, only the amount of positive tumor cells were scored, tumor proportion score (TPS). Of note, membrane as well as cytoplasmatic staining was assessed as positive. Secondly, the immune related cells comprising macrophages and lymphocytes were assessed, immune cell score (ICS). Finally, a combined positive score (CPS) of both was calculated. For all scores, the amount of posi- tive cells was calculated in relation to tumor cells multiplied by 100 . All calculations were made on 20-fold magnification.

\section{Statistical analysis}

Statistical analysis was performed using performed using GraphPad Prism 5 (GraphPad Software, La Jolla, CA, USA). Collected data were evaluated by means of descriptive statistics. Mean values were stated with standard deviation in all instances. Spearman's correlation coefficient (r) was used to analyze associations between investigated parameters. A two-sided Mann-Whitney-Test was used to test between groups. P-values $<0.05$ were taken to indicate statistical significance.

\section{Results}

Table 2 summarizes the investigated ADC histogram parameters.

For the PD-L1 expression of the TPSa mean value of $3.1 \pm 14.8$ was identified. Overall, 23 tumors (79.3\% of all patients) showed no expression.

Furthermore, for the PD-L1 expression of the CPS a mean value of $4.7 \pm 14.0$ was identified and 13 tumors (45.0\%) showed no expression.

Finally, for the PD-L1 expression of the ICS a mean value of $2.5 \pm 3.8$ was identified. 13 patients $(45.0 \%)$ showed no expression.

TABLE 2. Overview of the investigated apparent diffusion coefficient (ADC) histogram parameters

\begin{tabular}{ccc}
\hline Parameter & $\begin{array}{c}\text { Mean } \pm \text { standard } \\
\text { deviation, } \times 10^{-3} \mathrm{~mm}^{2} / \mathrm{s}\end{array}$ & $\begin{array}{c}\text { Range, } \\
\mathbf{x} 10^{-3} \mathrm{~mm}^{2} / \mathrm{s}\end{array}$ \\
\hline Mean & $1.14 \pm 0.22$ & $0.78-1.68$ \\
Min & $0.70 \pm 0.25$ & $0.17-1.24$ \\
Max & $1.78 \pm 0.32$ & $1.35-2.39$ \\
\hline P10 & $0.90 \pm 0.21$ & $0.54-1.41$ \\
\hline P25 & $1.0 \pm 0.21$ & $0.64-1.50$ \\
\hline P75 & $1.27 \pm 0.24$ & $0.87-1.82$ \\
\hline P90 & $1.41 \pm 0.26$ & $0.94-2.03$ \\
\hline Median & $1.12 \pm 0.22$ & $0.76-1.64$ \\
\hline Mode & $0.97 \pm 0.28$ & $0.78-1.55$ \\
\hline Kurtosis & $3.74 \pm 1.40$ & $2.23-7.93$ \\
Skewness & $0.49 \pm 0.49$ & $-0.54-1.49$ \\
Entropy & $2.44 \pm 0.49$ & $1.67-3.75$ \\
\hline
\end{tabular}

$P=$ percentile 

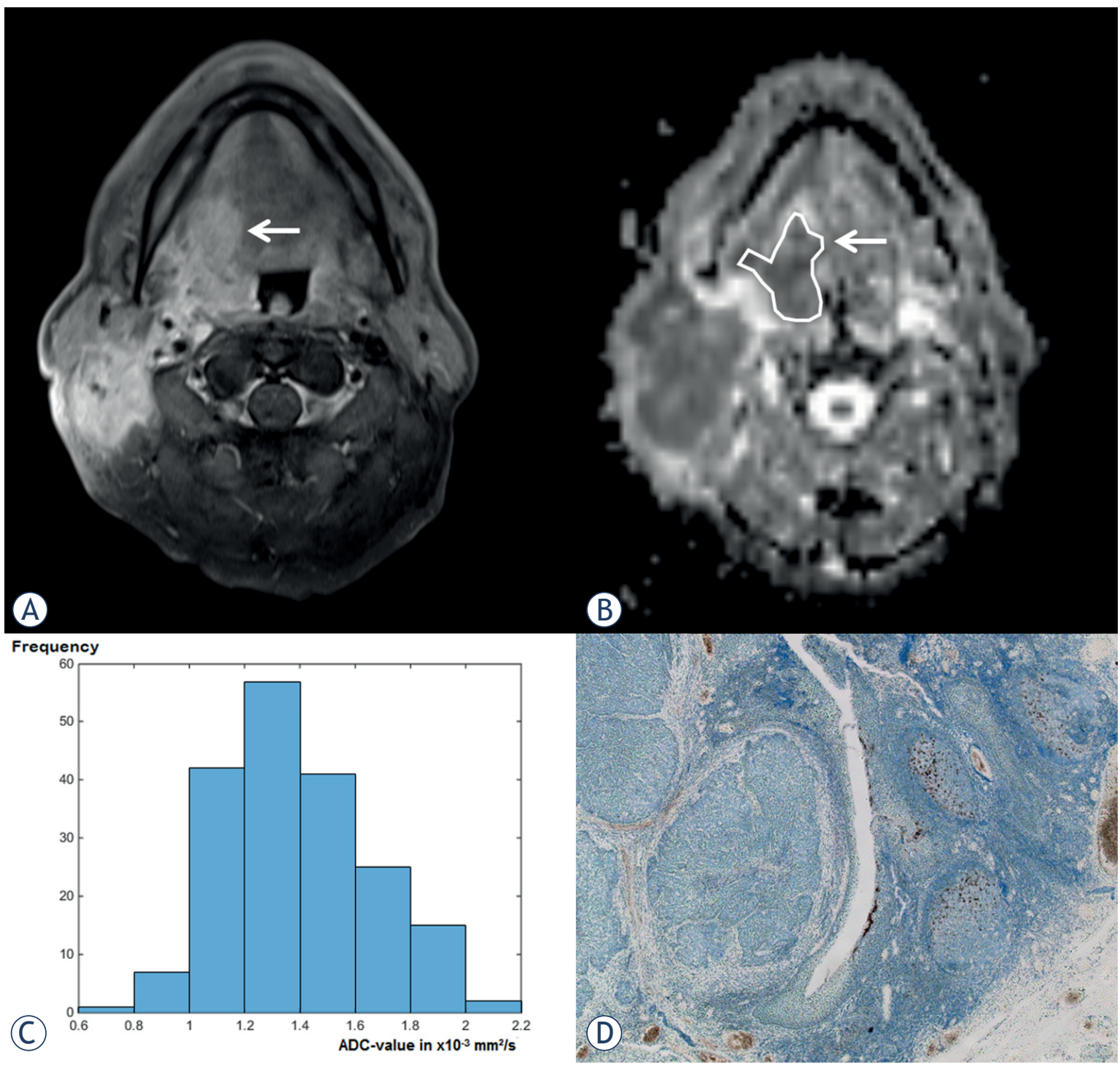

FIGURE 1. Representative case with a negative programmed cell death ligand I (PD-LI) score. (A) Postcontrast Tl-weighted image shows a large enhancing tumor of the right tongue base (arrow). A large lymph node metastasis on the right side can also be appreciated. (B) Apparent diffusion coefficient (ADC) map of the same slide. Both lesions show a marked hypointensity consistent with a diffusion restriction. The tumor (arrow) is surrounded with a region of interest, which is drawn on every tumor showing slide within the margins of the tumor in accordance to the corresponding Tl-weighted images. (C) The resulting ADC histogram of the tumor. The histogram analysis parameters $\left(\times 10^{-3} \mathrm{~mm}^{2} / \mathrm{s}\right)$ are as follows: $\mathrm{ADCmin}=0.79, \mathrm{ADC}$ mean $=1.38, \mathrm{ADC} \mathrm{max}=2.05, \mathrm{P} 10$ $=1.05$, P25 $=1.19$, P75 $=1.53$, P90 $=1.78$, median $=1.35$, mode $=1.27$, kurtosis $=2.61$, skewness $=0.41$ and entropy $=2.44$. (D) The corresponding PD-L1 stained specimen. No staining can be appreciated resulting in a value of 0 in all calculated scores.

There was a moderate correlation between TPS and CPS $(r=0.57, p=0.0015)$ and ICS $(r=0.57, p=$ $0.016)$. There was a strong correlation between ICS and CPS $(r=0.95, p<0.001)$.

Regarding associations between ADC histogram parameters and PD-L1-expression scores, there was only one weak correlation identified between skewness and ICS $(r=-0.38, p=0.04)$. ADC $C_{\max }$ tended to correlate with ICS $(r=-0.35, p=0.06)$. For TPS, the best association was identified withADCp25 $(\mathrm{r}=-0.36, \mathrm{p}=0.052)$.

In discrimination analysis there were no significant differences between TPS negative and positive tumors in regard of ADC histogram parameters.

In tumors with high expression of ICS (5-15 points) skewness was significantly lower than in lesions without ICS expression, $0.09 \pm 0.26$ vs. 0.56 $\pm 0.51(\mathrm{p}=0.025)$, respectively. Other parameters did not differ significantly. 


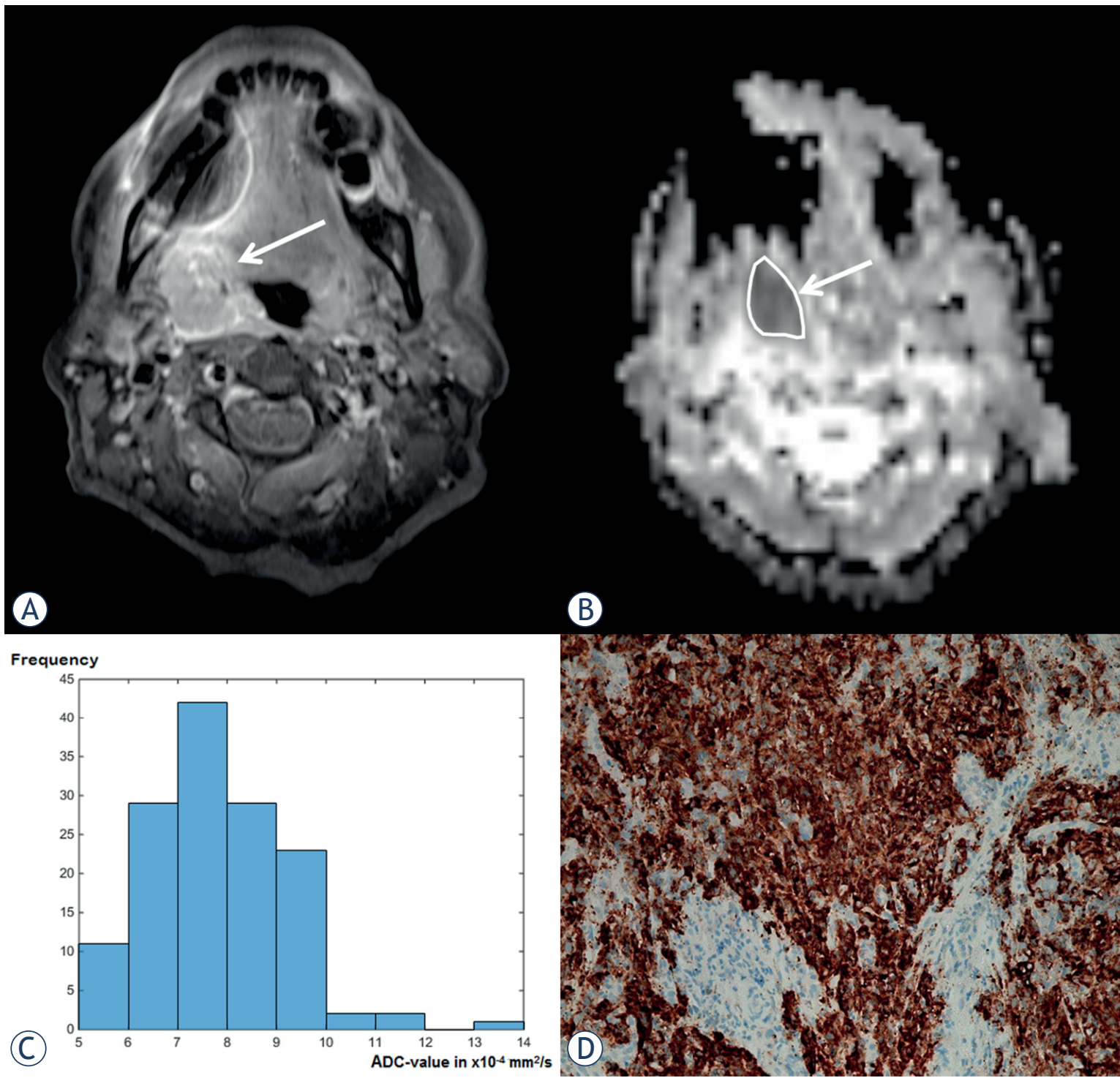

FIGURE 2. Representative case with a positive programmed cell death ligand 1 (PD-L1) score. (A) On the postcontrast Tl-weighted image a large enhancing tumor of the side within the right palatine tonsil (arrow). Susceptibility artifacts due to dental material can also be appreciated. (B) Apparent diffusion coefficient (ADC) map on the corresponding slide. A marked hypointensity can be seen consistent with a diffusion restriction. (C) The resulting ADC histogram of the tumor. The histogram analysis parameters $\left(\times 10^{-3}\right.$ $\mathrm{mm}^{2} / \mathrm{s}$ ) are as follows: $\mathrm{ADCmin}=0.51, \mathrm{ADCmean}=0.78, \mathrm{ADCmax}=1.36, \mathrm{P} 10=0.63, \mathrm{P} 25=0.69, \mathrm{P} 75=0.87, \mathrm{P} 90=0.94$, median $=0.76$, mode $=0.69$, kurtosis $=4.57$, skewness $=0.72$, and entropy $=2.41$. (D) The corresponding PD-L1 stained specimen. A weak staining of PD-L1 for tumor cells and associating immune cells can be appreciated. The resulting score values are 5 for the combined positive score and 3 for the immune cell score.

\section{Discussion}

This study sought to elucidate associations between DWI and PD-L1-expression in HNSCC. As shown, a moderate association between skewness derived from ADC values and the immune cell score was identified.

There is increasing body of evidence that different ADC values are capable to reflect tumor micro- structure..$^{3-5}$ Thus, results pooled out of many tumor entities suggests that there is an overall moderate inverse correlation between ADC values and tumor cellularity. ${ }^{5}$

Beyond that, there are several studies, which emphasized the fact that ADC values can also predict several biological features of tumors, such as neoangiogenesis and proliferation potential.,46-8 Regarding proliferation potential, an overall mod- 


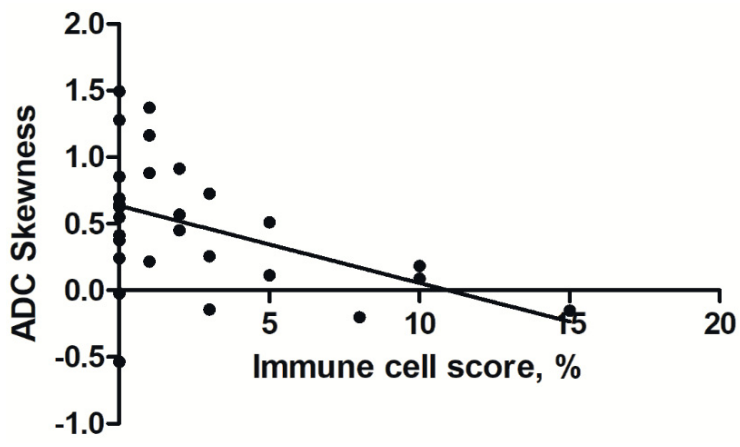

FIGURE 3. Correlation analysis between skewness derived from apparent diffusion coefficient (ADC) and the immune cell score (ICS). The resulting Spearman's correlation coefficient is $r=-0.38, p=0.04$.

erate inverse correlation was identified between ADC values and $\mathrm{Ki} 67$ index pooled for various tumors ${ }^{21}$ indicating that ADC can predict the amount of proliferating cells in tissue.

Regarding HNSCC, the relationship between ADC values and Ki 67 index was also identified ( $\mathrm{r}$ $=-0.61) .{ }^{4}$ In another study it was reported that ADC values were associated with CD3 positive stained cells $(r=-0.568, p=0.009)$, which is a measurement of the influx of T-cells within the tumor. ${ }^{7}$ Therefore, it can be assumed that ADC values cannot only reflect the whole cell density within the tumor but are sensitive to smaller specific tumor cell fractions. Interestingly, skewness was only associated with the amount of positive PD-L1-stained immune cells. Taken together with the results that ADC values were associated with the amount of tumor invading T-cells within the tumor, there might be a possibility to reflect and predict tumor microenvironment in HNSCC.

The parameter skewness represents the asymmetry of the distribution within the histogram in regard of the ADC values. ${ }^{22}$ In short, tumors which lean to the left have consecutively lower ADC values and thus a higher malignancy, whereas tumors, which lean to the right have higher ADC values tumors and thus lower malignancy. ${ }^{22}$ Regarding HNSCC, skewness was significantly higher in patients with HPV-mediated oropharyngeal cancer. ${ }^{23}$ Interestingly, there is an association between positive human papilloma patients and PD-L1 expression. ${ }^{18}$ This might explain why skewness derived from ADC values is related to both histopathological features of HNSCC.

In a recent excellent investigation, a direct correlation between PET-MRI and immunohistochemical analysis was performed based upon postopera- tive MRI scans with direct pathology coregistration. ${ }^{24}$ Furthermore, ADC correlated well with the apoptotic marker p53, Ki67, and with the apoptotic biomarker Bcl-2. Interestingly, ADC correlated also with expression of PD-L1. ${ }^{24}$

Also, ADC related parameters significantly correlated with the proportion of tumor stroma. ${ }^{25}$

In short, there is preliminary data to elucidate the possibility for imaging modalities to predict histopathology features for better patient guidance. More studies are definitely needed for better understanding of these complex interactions.

Recently, there is extensive interest about PD-1and PD-L1-inhibitors as a form of immunotherapy in oncology. ${ }^{16,17}$ In melanoma patients, PD-1 and PD-L1 inhibitors showed the first very promising results. The effectiveness of this immunotherapy was shortly after also shown for non-small lung cancer and other tumor entities. ${ }^{17}$

Regarding HNSCC, there were several trials establishing the effectiveness of this novel treatment in the recurrent setting. ${ }^{26-28}$ In these trials, PD-L1 status was defined as negative or positive with at least $1 \%$ of positive staining, well comparable with the approach in the present study.

In one study the probability of response was not significantly different between PD-L1 negative and positive patients, when only tumor cells were calculated but only when immune cells were also included into the analysis. ${ }^{28}$ Correspondingly to our results, skewness was only significantly associated with the scoring system regarding immune cells, reflecting these small, yet not negligible differences in PD-L1 staining scores. As shown in our results, there are significant differences between the scores, which needs to be considered. The immune cell score only measures the tumor associated PD-L1 cells and not the expression of the cancer cells themselves.

Moreover, it is well known that HNSCC is a highly heterogeneous tumor with different biological behavior comprising different localizations andHPV-mediated oropharyngeal cancer. ${ }^{17}$

Beyond that, even intratumoral heterogeneity was described as very important in HNSCC, which the amount of heterogeneity differing between primary localization. ${ }^{29}$ This might be important for biopsy sampling, as the tumor might significantly show different PD-L1 expression in several biopsy sites. This is, moreover, of special interest, as PD-L1 expression in head and neck cancer is localized at the tumor borders in HPV-positive cancers. ${ }^{30}$ Presumably, only imaging with a quantitative analysis approach can display this tumor 
heterogeneity completely during clinical routine. There might also be the opportunity for ADC histogram parameters to indicate treatment changes of the tumors during PD-L1 treatment.

To date, only one other study investigated possible associations between ADC values and PD-L1 expression in other tumors in oncology. ${ }^{31}$ In this study, there was a reported statistical difference between PD-L1 positive compared to negative tumors in regard of ADCmax in rectal cancers. ${ }^{31}$ Correspondingly, there was a statistical trend in the present study for ADCmax to correlate with the ICS, but it did not reach statistical significance, presumably due to small sample size. More studies are needed to investigate, whether ADCmax might be a promising biomarker to predict PD-L1 expression in oncologic imaging.

To overcome this limitation, further studies are needed with a multiparametric approach including MRI, CT and PET data guided with artificial intelligence. Presumably, non-invasive prediction of histopathology by imaging can be translated into clinical routine employing this approach.

There are several limitations of the present study to address. First, it is a retrospective analysis performed on a prospectively recruited patient sample. However, the imaging and pathology analyses were performed independently to each other to reduce possible bias. Second, the imaging analysis was performed as a whole tumor measurement, whereas the PD-L1analysis was performed on bioptic specimens with possible spatial incongruencies. Yet, this approach represents daily clinical practice for which no whole lesion pathologyradiology correlation is possible. Third, the ADC measurement was performed by one reader with possible accompanied reader-bias. However, it is reported that ADC values have a good to excellent interreader variability in HNSCC, which might reduce this limitation. Fourth, because of the exploratory study design we did not correct for multiple tests, which has an impact on the presented results. Fifth, the biopsy was taken from the primary tumor before the MRI. This might cause biopsy-related artifacts of the tumor, such as hematoma or local inflammation, which can have an impact on the ADC values.

In conclusion, here is a weak association between skewness derived from ADC values and PD-L1 expression in HNSCC, which might not be strong enough to predict PD-L1 expression in clinical routine. Presumably, ADC values are more influenced by complex histopathology compartments, comprising cellular and extracellular as- pects of tumors than only of a single subset of tumor associated cells.

\section{References}

1. McDermott JD, Bowles DW. Epidemiology of head and neck squamous cell carcinomas: impact on staging and prevention strategies. Curr Treat Options Oncol 2019; 20: 43. doi: 10.1007/s11864-019-0650-5

2. Widmann G, Henninger B, Kremser C, Jaschke W. MRI sequences in head \& neck radiology - state of the art. Rofo 2017; 189: 413-22. doi: 10.1055/s0043-103280

3. Padhani AR, Liu G, Koh DM, Chenevert TL, Thoeny HC, Takahara T, et al. Diffusion-weighted magnetic resonance imaging as a cancer biomarker: consensus and recommendations. Neoplasia 2009; 11: 102-25. doi: $10.1593 /$ neo. 81328

4. Surov A, Meyer HJ, Wienke A. Can imaging parameters provide information regarding histopathology in head and neck squamous cell carcinoma? A meta-analysis. Transl Oncol 2018; 11: 498-503. doi: 10.1016/j. tranon.2018.02.004

5. Surov A, Meyer $\mathrm{HJ}$, Wienke A. Correlation between apparent diffusion coefficient (ADC) and cellularity is different in several tumors: a meta-analysis. Oncotarget 2017; 8: 59492-9. doi: 10.18632/oncotarget.17752

6. Hauge A, Wegner CS, Gaustad JV, Simonsen TG, Andersen LMK, Rofstad EK. Diffusion-weighted MRI-derived ADC values reflect collagen I content in PDX models of uterine cervical cancer. Oncotarget 2017; 8: 105682-91. doi 10.18632/oncotarget. 22388

7. Swartz JE, Driessen JP, van Kempen PMW, de Bree R, Janssen LM, Pameije $\mathrm{FA}$, et al. Influence of tumor and microenvironment characteristics on diffusion-weighted imaging in oropharyngeal carcinoma: a pilot study. Oral Oncol 2018; 77: 9-15. doi: 10.1016/j.oraloncology.2017.12.001

8. Meyer HJ, Leifels L, Hamerla G, Höhn AK, Surov A. ADC-histogram analysis in head and neck squamous cell carcinoma. Associations with different histopathological features including expression of EGFR, VEGF, HIF-1 $\alpha$, Her 2 and p53. A preliminary study. Magn Reson Imaging 2018; 54: 214-7. doi: 10.1016/j.mri.2018.07.013

9. Surov A. Meyer HJ, Winter K, Richter C, Hoehn AK. Histogram analysis parameters of apparent diffusion coefficient reflect tumor cellularity and proliferation activity in head and neck squamous cell carcinoma. Oncotarget 2018; 9: 23599-607. doi: 10.18632/oncotarget.25284

10. Driessen JP, van Kempen PM, van der Heijden GJ, Philippens ME, Pameijer FA, Stegeman I, et al. Diffusion-weighted imaging in head and neck squamous cell carcinomas: a systematic review. Head Neck 2015; 37: 440-8. doi: 10.1002/hed.23575

11. Koontz NA, Wiggins RH 3rd. Differentiation of benign and malignant head and neck lesions with diffusion tensor imaging and DWI. AJR Am J Roentgenol 2017; 208: 1110-5. doi: 10.2214/AJR.16.16486

12. Surov A. Meyer HJ, Wienke A. Apparent diffusion coefficient for distinguishing between malignant and benign lesions in the head and neck region: a systematic review and meta-analysis. Front Oncol 2020; 9: 1362. doi: 10.3389/fonc. 2019.01362

13. King AD, Chow KK, Yu KH, Mo FK, Yeung DK, Yuan J, et al. Head and neck squamous cell carcinoma: diagnostic performance of diffusion-weighted MR imaging for the prediction of treatment response. Radiology 2013; 266: 531-8. doi: 10.1148/radiol.12120167

14. Law BK, King AD, Bhatia KS, Ahuja AT, Kam MK, Ma BB, et al. Diffusionweighted imaging of nasopharyngeal carcinoma: can pretreatment DWI predict local failure based on long-term outcome? AJNR Am J Neuroradiol 2016; 37: 1706-12. doi: 10.3174/ajnr.A4792.

15. Matoba M, Tuji H, Shimode Y, Toyoda I, Kuginuki Y, Miwa K, et al. Fractional change in apparent diffusion coefficient as an imaging biomarker for predicting treatment response in head and neck cancer treated with chemoradiotherapy. AJNR Am J Neuroradiol 2014; 35: 379-85. doi: 10.3174/ajnr. A3706

16. Schneider S, Kadletz L, Wiebringhaus R, Kenner L, Selzer E, Füreder T, et al PD-1 and PD-L1 expression in HNSCC primary cancer and related lymph node metastasis - impact on clinical outcome. Histopathology 2018; 73: 573-84. doi: $10.1111 /$ his.13646 
17. Leemans CR, Snijders PJF, Brakenhoff RH. The molecular landscape of head and neck cancer. Nat Rev Cancer 2018; 18: 269-82. doi: 10.1038/ nrc.2018.11

18. Tang H, Zhou X, Ye Y, Zhou Y, Wu C, Xu Y. The different role of PD-L1 in head and neck squamous cell carcinomas: a meta-analysis. Pathol Res Pract 2020; 216: 152768. doi: 10.1016/j.prp.2019.152768

19. Yang WF, Wong MCM, Thomson PJ, Li KY, Su YX. The prognostic role of PD-L1 expression for survival in head and neck squamous cell carcinoma: a systematic review and meta-analysis. Oral Oncol 2018; 86: 81-90. doi: 10.1016/j.oraloncology.2018.09.016

20. Lin YM, Sung WW, Hsieh MJ,Tsai SC, Lai HW, Yang SM, et al. High PD-L1 expression correlates with metastasis and poor prognosis in oral squamous cell carcinoma. PLoS One 2015; 10: e0142656. doi: 10.1371/journal. pone.0142656.

21. Surov A, Meyer HJ, Wienke A. Associations between apparent diffusion coefficient (ADC) and KI 67 in different tumors: a meta-analysis. Part 1: $A D C_{\text {mean. }}$ Oncotarget 2017; 8: 75434-44. doi: 10.18632/oncotarget.20406

22. Just N. Improving tumour heterogeneity MRI assessment with histograms. Br J Cancer 2014; 111: 2205-13. doi: 10.1038/bjc.2014.512

23. de Perrot T, Lenoir V, Domingo Ayllón M, Dulguerov N, Pusztaszeri M, Becker M. Apparent diffusion coefficient histograms of human papillomaviruspositive and human papillomavirus-negative head and neck squamous cell carcinoma: assessment of tumor heterogeneity and comparison with histopathology. AJNR Am J Neuroradiol 2017; 38: 2153-60. doi: 10.3174/ ajnr.A5370.

24. Rasmussen $\mathrm{JH}$, Olin A, Lelkaitis $\mathrm{G}$, Hansen $\mathrm{AE}$, Andersen $\mathrm{FL}$, Johannesen $\mathrm{HH}$, et al. Does multiparametric imaging with ${ }^{18}$ F-FDG-PET/MRI capture spatial variation in immunohistochemical cancer biomarkers in head and neck squamous cell carcinoma? Br J Cancer 2020; 123: 46-53. doi: 10.1038/ s41416-020-0876-9

25. Choi JW, Lee D, Hyun SH, Han M, Kim JH, Lee SJ. Intratumoural heterogeneity measured using FDG PET and MRI is associated with tumour-stroma ratio and clinical outcome in head and neck squamous cell carcinoma. Clin Radiol 2017; 72: 482-9. doi: 10.1016/j.crad.2017.01.019

26. Chow LQM, Haddad R, Gupta S, Mahipal A, Mehra R, Tahara M, et al. Antitumor activity of pembrolizumab in biomarker-unselected patients with recurrent and/or metastatic head and neck squamous cell carcinoma: results from the phase Ib KEYNOTE-012 expansion cohort. J Clin Oncol 2016; 34: 3838-45. doi: 10.1200/JCO.2016.68.1478.

27. Ferris RL, Blumenschein G Jr, Fayette J, Guigay J, Colevas AD, Licitra L, et al. Nivolumab for recurrent squamous-cell carcinoma of the head and neck. $N$ Eng/ J Med 2016; 375: 1856-67. doi: 10.1056/NEJMoa1602252

28. Seiwert TY, Burtness B, Mehra R, Weiss J, Berger R, Eder JP, et al. Safety and clinical activity of pembrolizumab for treatment of recurrent or metastatic squamous cell carcinoma of the head and neck (KEYNOTE-012): an openlabel, multicentre, phase $1 \mathrm{~b}$ trial. Lancet Oncol 2016; 17: 956-65. doi: 10.1016/S1470-2045(16)30066-3

29. Ledgerwood LG, Kumar D, Eterovic AK, Wick J, Chen K, Zhao H, et al. The degree of intratumor mutational heterogeneity varies by primary tumo sub-site. Oncotarget 2016; 7: 27185-98. doi: 10.18632/oncotarget.8448.

30. Lyford-Pike S, Peng S, Young GD, Taube JM, Westra WH, Akpeng B, et al. Evidence for a role of the PD-1: PD-L1 pathway in immune resistance of human papillomavirus -associated head and neck squamous cell carcinoma. Cancer Res 2013; 73: 1733-41. doi: 10.1158/0008-5472.CAN-12-2384.

31. Meyer HJ, Höhn A, Surov A. Histogram analysis of ADC in rectal cancer: associations with different histopathological findings including expression of EGFR, Hif1-alpha, VEGF, p53, PD1, and KI 67. A preliminary study. Oncotarget 2018; 9: 18510-7. doi: 10.18632/oncotarget.24905 